The Astronomical Journal, 133:2389-2392, 2007 May

(C) 2007. The American Astronomical Society. All rights reserved. Printed in U.S.A

\title{
THE SELF-SIMILARITY OF SHEAR-DOMINATED VISCOUS STIRRING
}

\author{
Benjamin F. Collins, Hilke E. Schlichting, and Re’em Sari \\ California Institute of Technology, Pasadena, CA 91125, USA; bfc@tapir.caltech.edu \\ Received 2006 September 21; accepted 2007 February 8
}

\begin{abstract}
We examine the growth of eccentricities of a population of particles with nearly circular orbits around a central massive body. Successive encounters between pairs of particles increase the eccentricities in the disk on average. We describe the system in terms of a Boltzmann equation. As long as the epicyclic motions of the particles are small compared to the shearing motion between circular Keplerian orbits, there is no preferred scale for the eccentricities, and the evolution is self-similar. This simplification reduces the full time-dependent Boltzmann equation to two separate equations: one that describes the shape of the distribution and another that describes the evolution of the characteristic eccentricity on which the distribution is centered. We find that the shape of the eccentricity distribution function is a general feature of such systems, and is of the form $\left(1+x^{2}\right)^{-3 / 2}$. In particular, bodies evolving under only their own excitations have the same eccentricity distribution profile as bodies whose excitations are balanced by dynamical friction. We find exact expression for the typical eccentricity for these two cases, and allow for time-dependent damping and excitation rates. Full numerical $N$-body simulations of a disk with 200 planetesimals verify our analytical selfsimilar distribution.
\end{abstract}

Key words: planets and satellites: formation — solar system: formation

\section{INTRODUCTION}

Modern computational power allows the simultaneous integration of the orbits of increasingly numerous particles. Much of the planet formation process, however, involves particle numbers that exceed the limits of computational efficiency. This limitation is often circumvented with a statistical approach. By monitoring the gravitational interactions of the particles in a timeaveraged sense, various properties of the particle population can be calculated without a full $N$-body simulation.

Collins \& Sari (2006, hereafter Paper I) motivate a Boltzmann equation to describe the evolution of the eccentricity distribution of an ensemble of particles in which the relative motion between any two interacting particles is dominated by the shearing motion of close circular orbits. Such a regime of orbital eccentricities is called shear-dominated. The solution of their equation provides a simple analytic expression for the equilibrium eccentricity distribution that results when dynamical friction can balance the mutual interactions of the particles; the analytic expression matches results from numerical simulations remarkably well.

In this paper we derive analytically the nonequilibrium distribution function of interacting shear-dominated particles in the absence of dynamical friction. Section 2 reviews the construction of the Boltzmann equation. In $\S 3$ we show that the distribution function behaves self-similarly and that the shape of the nonequilibrium distribution function is identical to the equilibrium distribution of Paper I. Section 4 generalizes our Boltzmann equation and its solution to include time-dependent rates of excitation and eccentricity-damping interactions. Section 5 corroborates our analytical results with numerical simulations. Conclusions follow in $\S 6$.

\section{THE TIME-DEPENDENT BOLTZMANN EQUATION}

We consider a disk of particles on initially circular orbits around a massive central body. We write their surface mass density $\Sigma$ and the mass of a single body $m$. The number density $\Sigma / m$ is sufficiently low that three-body encounters are very rare; therefore the orbital evolution of each body is well described as a sequence of pairwise encounters.

The change in eccentricity due to one such encounter can be calculated analytically. For completeness, we summarize the derivation presented in Paper I. Let one particle, with a semimajor axis $a$, encounter another with semimajor axis $a+b$. In the limit of $b \ll a$, the relative orbital frequency between the pair is $\Omega_{r}=$ $(3 / 2) \Omega b / a$, where $\Omega$ is the Keplerian orbital frequency for a semimajor axis $a$. If in addition $b \gg R_{\mathrm{H}} \approx\left[m /\left(3 M_{\odot}\right)\right]^{1 / 3} a$, the change in eccentricity from one encounter is $e_{k}=\left(A_{k} / 3\right)\left(m / M_{\odot}\right)(b / a)^{-2}$, where $A_{k} \approx 6.67$ collects the order-unity coefficients (Goldreich $\&$ Tremaine 1978; Petit \& Henon 1986).

The eccentricity is not the only Keplerian element that characterizes the noncircular motion of a particle; the longitude of periapse specifies the relative orientation of a particle's epicycle. The particles may also follow orbits that do not lie in the disk. However, shear-dominated viscous stirring excites inclinations at a rate that is always slower than the excitation of eccentricities (Wetherill \& Stewart 1993; Goldreich et al. 2004; Rafikov 2003). The perpendicular velocities are, in this case, always negligible compared to the epicyclic motion in the disk plane.

The magnitude of an orbit's eccentricity and the longitude of periapse together specify a two-dimensional parameter space. We describe the two-dimensional variable with a vector, $\boldsymbol{e}=$ $\{e \cos \omega, e \sin \omega\}$. The distribution function is a function of this vector and time, $f(\boldsymbol{e}, t)$. That the changes in $\boldsymbol{e}$ due to encounters do not depend on the longitude of periapse already shows that the distribution function must be axisymmetric, or $f(\boldsymbol{e}, t)=$ $f(e, t)$. Then the number of bodies per unit logarithmic interval around $e$ is given by $2 \pi e^{2} f(e, t)$.

We characterize the eccentricity growth with a differential rate, $p\left(\boldsymbol{e}_{k}\right) d^{2} \boldsymbol{e}_{k}$, that the eccentricity vector of a particle will be changed by an amount $\boldsymbol{e}_{k}$. Since the change in eccentricity experienced by a pair of bodies, when treated as a vector quantity, is independent of the initial eccentricity vector of each body, this function is also axisymmetric and only depends on the magnitude of the change of eccentricity, $e_{k}$. 
The excitation rate depends on the surface mass density of particles in the disk, $\Sigma$, the mass of a single body, $m$, the mass of the central star, $M_{\odot}$, the cross section at which a particle experiences encounters of a strength $e_{k}$, and the relative speed of those encounters. The impact parameter at which a particle receives an eccentricity $e_{k}$ scales as $b \propto e_{k}^{-1 / 2}$. If the eccentricities are small, the speed at which one particle encounters the others is set only by the shearing of their two orbits, which is proportional to $b$. Then, as shown in Paper I,

$$
2 \pi p\left(e_{k}\right) e_{k} d e_{k}=3 \frac{\Sigma}{m} \Omega b\left(e_{k}\right) d b\left(e_{k}\right)
$$

After simplification, we find

$$
p\left(e_{k}\right)=\frac{A_{k}}{4 \pi} \frac{\Sigma a^{2}}{M_{\odot}} \frac{1}{e_{k}^{3}} \Omega .
$$

An integral over every $e_{k}$ dictates the rate of change of the number of bodies with a given eccentricity, $e$ :

$$
\frac{\partial f(e, t)}{\partial t}=\iint p\left(\left|\boldsymbol{e}-\boldsymbol{e}_{n}\right|\right)\left[f\left(e_{n}, t\right)-f(e, t)\right] d^{2} \boldsymbol{e}_{n} .
$$

Note that this equation implicitly conserves the total particle number, $\iint f(e, t) d^{2} \boldsymbol{e}=1$. This can be shown by integrating both sides with respect to $\boldsymbol{e}$.

\section{THE SELF-SIMILAR DISTRIBUTION}

Without a specific eccentricity scale to dictate the evolution of $f(e, t)$, we expect a solution of the form

$$
f(e, t)=F(t) g\left(e / e_{c}(t)\right) .
$$

Replacing $f(e, t)$ in equation (3) with equation (4), we find

$$
\begin{aligned}
& \frac{1}{F(t)} \frac{d F(t)}{d t} e_{c}(t) g(x)-x \frac{d g(x)}{d x} \dot{e}_{c}(t) \\
= & \iint p\left(\left|\boldsymbol{x}-\boldsymbol{x}_{n}\right|\right)\left[g\left(x_{n}\right)-g(x)\right] d^{2} \boldsymbol{x}_{n},
\end{aligned}
$$

where $\boldsymbol{x}=\boldsymbol{e} / e_{c}(t)$. The additional constraint that equation (3) conserves particle number implies $F(t) e_{c}(t)^{2}$ is constant. This relationship simplifies the left side of equation (5) such that the only possible time dependence of each term is contained in $\dot{e}_{c}(t)$. The right-hand side, however, is independent of time. Therefore $\dot{e}_{c}(t)$ must be constant. Then,

$$
e_{c}(t)=C_{e} t, \quad F(t)=\left(C_{e} t\right)^{-2} .
$$

The overall normalization of $F(t)$ is arbitrary, as it can be absorbed into $g(x)$. Our choice of $F(t)$ requires $\iint g(x) d^{2} \boldsymbol{x}=1$ to ensure that $\iint f(e, t) d^{2} \boldsymbol{e}=1$ for all $t$. Physically, the typical eccentricity, $e_{c}(t)$, is set by the eccentricity change that occurs once per particle per time $t$, or, $e_{c}(t)^{2} p\left(e_{c}(t)\right) t \sim 1$. This argument sets $e_{c}(t)$ only up to a constant coefficient; for simplicity we choose the coefficients such that

$$
e_{c}(t)=\frac{A_{k}}{2} \frac{\Sigma a^{2}}{M_{\odot}} \Omega t
$$

Previous order-of-magnitude arguments by Goldreich et al. (2004) also show this scaling with $\Sigma$ and $t$.
Finally, the profile shape, $g(x)$, is specified by the integrodifferential equation

$$
2 g(x)+x \frac{d g(x)}{d x}+\frac{1}{2 \pi} \iint \frac{g\left(x_{n}\right)-g(x)}{\left|\boldsymbol{x}_{n}-\boldsymbol{x}\right|^{3}} d^{2} \boldsymbol{x}_{n}=0 .
$$

Equation (8) is identical to equation (17) of Paper I. A detailed description of the equation and its solution can be found in that paper. We present here a simpler and more direct derivation of the solution. We define the two-dimensional Fourier transform of $g(x)$ as $G(\boldsymbol{k})=\int g(x) e^{i \boldsymbol{k} \cdot \boldsymbol{x}} d^{2} \boldsymbol{x}$. Since $g(x)$ is isotropic, $G(\boldsymbol{k})=$ $G(k)$ with $0<k<\infty$. By taking the two-dimensional Fourier transform of equation (8), we find $d G(k) / d k=-G(k)$. This simple differential equation is satisfied by $G(k)=e^{-k}$. The boundary condition $G(0)=1$ is equivalent to our normalization of $g(x)$. The solution $g(x)$ is then

$$
g(x)=\frac{1}{2 \pi}\left(1+x^{2}\right)^{-3 / 2} .
$$

\section{THE GENERALIZED TIME-DEPENDENT DISTRIBUTION}

In addition to the eccentricity excitation mechanism discussed in $\S 2$, it is reasonable to include a dissipative term in the Boltzmann equation that accounts for processes that reduce the eccentricities of the bodies. One relevant example of such a process is the dynamical friction caused by a collection of small bodies. The analysis of Paper I examines the limit in which this dissipation balances mutual excitation: the distribution is in the shape of equation (9), and its characteristic eccentricity does not evolve with time. Section 3 can be considered the complementary limit in which the stirring term dominates the whole expression: the distribution of equation (9) increases linearly with time according to equation (6). In this section we derive how the characteristic eccentricity evolves as a function of time in between these two regimes.

The full time-dependent Boltzmann equation (see Paper I) is

$$
\begin{aligned}
\frac{\partial f(e, t)}{\partial t}= & \frac{2 f(e, t)}{\tau_{d}}+\frac{e}{\tau_{d}} \frac{\partial f(e, t)}{\partial e} \\
& +\iint p\left(\left|\boldsymbol{e}-\boldsymbol{e}_{n}\right|\right)\left[f\left(e_{n}, t\right)-f(e, t)\right] d^{2} \boldsymbol{e}_{n} .
\end{aligned}
$$

We substitute equation (4) for $f(e, t)$ and retain the condition $\iint g(x) d^{2} \boldsymbol{x}=1$, which dictates that $F(t)=e_{c}(t)^{-2}$. For clarity, we also separate the eccentricity dependence from the excitation term: $p(e) \equiv C e^{-3}$. This constant $C$ collects the coefficients that can be read from equation (2). Then equation (10) becomes

$$
\begin{aligned}
0= & \frac{1}{C}\left[\frac{e_{c}(t)}{\tau_{d}}+\dot{e}_{c}(t)\right]\left(2 g(x)+x \frac{d g(x)}{d x}\right) \\
& +\iint \frac{g\left(x_{n}\right)-g(x)}{\left|\boldsymbol{x}-\boldsymbol{x}_{n}\right|^{3}} d^{2} \boldsymbol{x}_{n} .
\end{aligned}
$$

To solve this equation for all $x$ and all $t$ simultaneously, the $x$ - and $t$-dependence must be the same for each term. Since $C$ and the terms in square brackets contain all of the time dependence, they must together be constant in time. We choose that constant to be $2 \pi$ so that the solution to this equation is consistent with our earlier definition of $g(x)$, equation (9). Then $e_{c}(t)$ obeys the ordinary differential equation:

$$
\dot{e}_{c}(t)+\frac{e_{c}(t)}{\tau_{d}}=2 \pi C .
$$




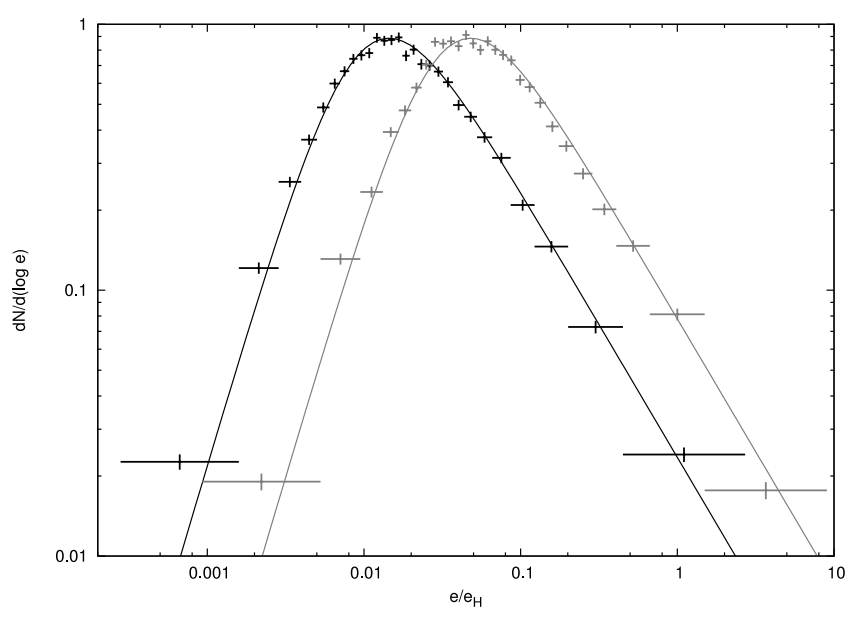

FIG. 1.-Eccentricity distributions of a shear-dominated disk of 200 particles each with a mass $m=5 \times 10^{-9} M_{\odot}$ after 3 (black line) and 10 (gray line) orbits. The average surface mass density of the simulated annulus is $3 \times 10^{-3} \mathrm{~g} \mathrm{~cm}^{-2}$. The vertical error bars are estimated by assuming each bin obeys Poisson statistics. The width of each bin has been chosen such that each bin contains a similar number of particles.

In the limit of no damping, or $\tau_{d} \rightarrow \infty$, we recover the $e_{c}(t)=$ $2 \pi C t$ relation found in $\S 3$. The time-independent characteristic eccentricity of Paper I is recovered by setting $\dot{e}_{c}(t)$ equal to zero, resulting in $e_{c}=2 \pi C \tau_{d}$. The two regimes are connected via the solution to equation $(12), e_{c}(t)=2 \pi C \tau_{d}\left[1-\exp \left(-t / \tau_{d}\right)\right]$.

Equation (12) can be rewritten in a more familiar form:

$$
\frac{1}{e_{c}(t)} \frac{d e_{c}(t)}{d t}=A_{k} \frac{9}{8 \pi} \frac{\Sigma \Omega}{\rho R} \alpha^{-2} \frac{e_{\mathrm{H}}}{e_{c}(t)}-\frac{1}{\tau_{d}}
$$

where $\alpha$ is the ratio of the planet's radius to its Hill sphere and $e_{\mathrm{H}}$ is the Hill eccentricity $\left(e_{\mathrm{H}}=R_{\mathrm{H}} / a\right)$. This equation, which now describes the rate at which $e_{c}(t)$ changes, is identical to the orderof-magnitude analysis reviewed in Goldreich et al. (2004). However, here we find the exact numerical coefficient for the excitation rate. We also give a definite meaning to $e_{c}(t)$ : it is the eccentricity scale of the distribution set by equation (9).

We note that the derivation of equation (13) does not require that $\Sigma, R$, and $\tau_{d}$ are constant. We can, in fact, apply the same Boltzmann equation to scenarios that include time-dependant excitation and damping rates. To demonstrate, we consider again the case of protoplanets embedded in a population of smaller planetesimals with a surface mass density of $\sigma$, and take into account the growth of the protoplanets from planetesimal accretion. Assuming that the eccentricities of the planetesimals and protoplanets are smaller than the Hill eccentricities of the protoplanets by more than $\alpha^{1 / 2}$, each protoplanet's radius grows at a rate (Dones \& Tremaine 1993)

$$
\frac{d R}{d t}=0.51 \frac{\sigma \Omega}{\rho} \alpha^{-3 / 2}
$$

For reference, the same planetesimals damp the eccentricities of the protoplanets at a rate (Goldreich et al. 2004)

$$
\frac{1}{\tau_{d}}=-\frac{1}{e} \frac{d e}{d t}=C_{d} \frac{\sigma \Omega}{\rho R} \alpha^{-2} .
$$

The best estimates for the numerical coefficient of the damping timescale are from $N$-body integrations; Ohtsuki et al. (2002) find a value of 1.2 .
We further assume that the planetesimal surface density is unaffected by the protoplanet accretion and that there are no collisions between the protoplanets. In this case $\Sigma$ and $e_{\mathrm{H}}$ depend on time only through the increasing radii of the protoplanets; given a constant $\dot{R}$, we have $\Sigma(t) \propto t^{3}$ and $e_{\mathrm{H}}(t) \propto t$. Therefore, the solution to equation (12) is

$$
e_{c}(t)=\left(1+\frac{4}{1.96 C_{d} \alpha^{-1 / 2}}\right)^{-1} \frac{A_{k}}{C_{d}} \frac{9}{8 \pi} \frac{\Sigma(t)}{\sigma} e_{\mathrm{H}}(t) .
$$

The dynamical friction rate is always faster than the growth rate of the protoplanets; thus this $e_{c}(t)$ is close to that of the equilibrium eccentricity distribution established at a constant $R$. The small fractional correction of $4 /\left(1.96 C_{d} \alpha^{-1 / 2}\right)$ accounts for the slight growth of each protoplanet over a damping timescale. Together with equation (9), equation (16) gives a remarkably simple analytic expression for the time-dependent eccentricity distribution of protoplanets that excite each other and are damped by a sea of planetesimals from which they also accrete.

\section{NUMERICAL SIMULATIONS}

The nonequilibrium distribution function of eccentricities in the shear-dominated regime can be measured directly from a full numerical simulation of the disk. We use a custom $N$-body integrator that evolves the changes in the two-body constants of motion of each particle around the central mass. These constants are chosen to vary slowly with small perturbations. Solving Kepler's equation for each body translates each time step into a change in orbital phase. The constants of motion are then integrated by a fourth-order Runge-Kutta routine with adaptive time steps (Press et al. 1992).

For this study we follow a disk of 200 equal-mass bodies, with $m=5 \times 10^{-9} M_{\odot}$, on initially circular orbits with randomly determined phases and semimajor axes within a small annulus of width $\Delta a=0.8 a$. To avoid possible artifacts from the edge of the simulation, we only measure the eccentricities of the bodies in the central third of the disk. A histogram of those eccentricities shows the number of bodies with each eccentricity, $e d N / d e$. To increase the signal-to-noise ratio of the histogram at each time, we add the results of 100 simulations with randomly generated initial semimajor axes and orbital phases.

Figure 1 shows the eccentricity distributions measured after 3 and 10 orbits. The horizontal error bars indicate the width of

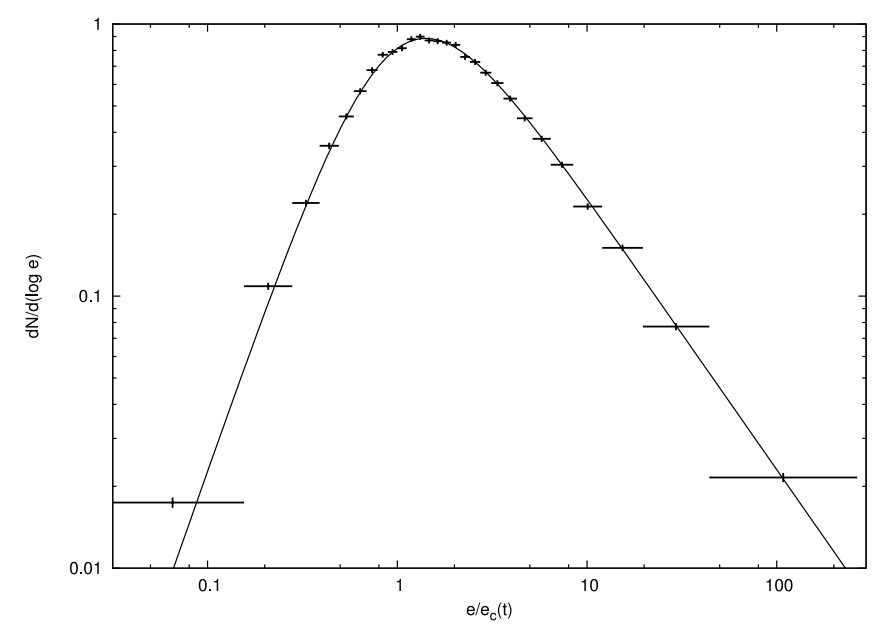

FIG. 2.-Eccentricity distribution of the same numerical simulation of Fig. 1. Here the distribution after 1,3 , and 10 orbits, scaled by the characteristic eccentricity at that time, are added together. The profile shape is very well described by eq. (9), plotted as a solid line. The error bars are assigned in the same way as in Fig. 1. 
each bin, and the vertical error bars are determined assuming that each bin is Poisson distributed. The analytic distribution function derived in $\S 3$ for each time is also plotted, as a solid line. The measured distributions agree remarkably with the analytic result.

To emphasize the self-similarity of the distribution shape, we scale the eccentricities measured at each time by the characteristic eccentricity at that time $\left[e_{c}(t)\right.$, given by eq. (6)] and plot the shapes added together. Figure 2 shows that the resulting distribution shape matches the analytic form of $g(x)$ very well.

\section{DISCUSSION}

We have written a time-dependent Boltzmann equation that describes the eccentricity distribution function of a population of orbiting particles under the influence of their mutual excitations in the shear-dominated regime. Reasoning that the distribution function of eccentricities should behave self-similarly, that is, retain a constant profile while its normalization and scaling depend on time, we have decoupled the time dependence of the eccentricity distribution from its shape. The shape is constant even in the presence of a dynamical-friction-like dissipation term. The evolution of the distribution in time can be determined generally with any time-dependent dynamical friction and excitation rates. Numerical experiments confirm this self-similarity and the analytic form of the distribution profile.

Although we have only considered disks of a single particle size, the formalism above applies trivially to disks with mass distributions. In fact, the characteristic eccentricity, equation (6), depends only on the total surface mass density of the disk. As long as bodies of every part of the mass spectrum are in the sheardominated regime, the eccentricities of all bodies are drawn from the same distribution. This is a consequence of the fact that gravitational acceleration is mass-independent. In contrast, the dynamical friction of Paper I depends on the size of each particle. The equilibrium distributions in that case do differ for each mass group.

Most of the disk bodies then have eccentricities of about $e_{c}(t)$. The mean eccentricity, $\iint e f(e, t) d^{2} \boldsymbol{e}$, is formally infinite; in reality the mean depends logarithmically on the maximum eccentricity achievable from one interaction. Higher moments of the distribution, such as $\left\langle e^{2}\right\rangle$, are dominated by the bodies with the maximum eccentricity. The random kinetic energy of the disk bodies, for example, is then set by the few bodies with the highest eccentricities regardless of the value of $e_{c}(t)$.

Since $e_{c}(t)$ is an increasing function of time, the condition of shear-dominated dynamics will be violated eventually. The interaction rate of the highest eccentricity bodies is low, so up to an order unity fraction of the bodies can have super-Hill eccentricities without affecting the shape of the sub-Hill distribution.
This assumes that the disk of protoplanets is uniform to the extent that every impact parameter up to the Hill radius is wellsampled by the interactions. Then, $e_{c}(t)$ grows linearly all the way to the Hill eccentricity. However, if the typical separation between bodies is substantially greater than a Hill radius, $b_{\min } \gg$ $R_{\mathrm{H}}$, then there is an upper limit in eccentricity that one interaction can provide: $e_{\max } \sim\left(b_{\min } / R_{\mathrm{H}}\right)^{-2} e_{\mathrm{H}}$. The assumptions of this work then break down as $e_{c}(t)$ approaches $e_{\max }$.

That the shape of the distribution function is identical in the limits of nonexistent or dominating dynamical friction is ultimately not surprising. In both cases the bodies in question excite their orbital parameters via the same shear-dominated viscous stirring mechanism. If dynamical friction is acting on these bodies, their eccentricities decrease with time proportionally to their magnitude. An equilibrium between excitations and this damping produces a characteristic eccentricity around which the eccentricities of all bodies are distributed. Without an agent of dynamical friction, the typical eccentricity of a body in the disk, $e_{\text {typical }} \sim e_{c}(t)$, grows with time. However, the ratio of the eccentricity of a particle that has not interacted recently, $e$, to that typical eccentricity shrinks proportionally to itself:

$$
\dot{x} \equiv \frac{d}{d t}\left(\frac{e}{e_{c}(t)}\right) \propto-x
$$

This is formally equivalent to the damping provided by the dynamical friction we describe here and in Paper I.

The physical scenario we have described in this work can be viewed as a particular case of systems that achieve steady states without reaching a thermodynamic equilibrium. A general model for such behavior is that of a "driven dissipative system": particles that collide inelastically with each other but also gain energy by interacting with an external reservoir. Analytic modeling of these systems with Boltzmann equations have found velocity distributions with the same properties that we presented here, namely non-Maxwellian profiles and self-similar evolution (Ben-Naim \& Krapivsky 2002; ben-Avraham et al. 2003; BenNaim \& Machta 2005). A more detailed study of the relationship between protoplanetary dynamics, these general models, and the physics that they represent may provide new perspectives for both fields.

We thank Yair Shokef for his enlightening comments, and the anonymous referee for several suggestions that have improved this manuscript. R. S. is a Packard Fellow and an Alfred P. Sloan Fellow.
ben-Avraham, D., Ben-Naim, E., Lindenberg, K., \& Rosas, A. 2003, Phys. Rev. E, 68, 050103

Ben-Naim, E., \& Krapivsky, P. L. 2002, Phys. Rev. E, 66, 011309

Ben-Naim, E., \& Machta, J. 2005, Phys. Rev. Lett., 94, 138001

Collins, B. F., \& Sari, R. 2006, AJ, 132, 1316 (Paper I)

Dones, L., \& Tremaine, S. 1993, Icarus, 103, 67

Goldreich, P., Lithwick, Y., \& Sari, R. 2004, ARA\&A, 42, 549

Goldreich, P., \& Tremaine, S. D. 1978, Icarus, 34, 227

\section{REFERENCES}

Ohtsuki, K., Stewart, G. R., \& Ida, S. 2002, Icarus, 155, 436

Petit, J.-M., \& Henon, M. 1986, Icarus, 66, 536

Press, W. H., Teukolsky, S. A., Vetterling, W. T., \& Flannery, B. P. 1992, Numerical Recipes in C: The Art of Scientific Computing (2nd ed.; Cambridge: Cambridge Univ. Press)

Rafikov, R. R. 2003, AJ, 125, 942

Wetherill, G. W., \& Stewart, G. R. 1993, Icarus, 106, 190 